\title{
graphed: A Web-Based Concept Mapping Application for Instruction and Research
}

\author{
Ioan G. Ionas ${ }^{1}$, Mugur V. Geana ${ }^{2}$ \\ ${ }^{1}$ Richard W. Riley College of Education, Walden University, USA, ${ }^{2}$ School of Journalism \\ and Mass Communication, Kansas University, USA.
}

\begin{abstract}
The ongoing worldwide pandemic has forced educational establishments to accelerate full-scale adoption of online learning at an accelerated pace, while the development of tools appropriate for remote instruction assessment is yet to catch up. Most of the time traditional assessment methods are still employed, but they are not always optimal for use in online environments; better tools are needed to help gain deeper insights into how students think and learn. graphed is a web application developed to support the assessment of learners' understanding and knowledge acquisition and, simultaneously, provide researchers with data that can help in the development of dedicated processes for the automatic evaluation and comparison of concept maps. Our goal is to take a more practical approach by studying the capabilities offered by existing software, libraries, and computational avenues to advance the use of concept maps as assessment tools. Preliminary findings suggest that the concept mapping activity has achieved its purpose of promoting deep thinking, that the application is relatively usable, and clarified the path for future development and enhancement. Examples on the use of graphed in the classroom are provided.
\end{abstract}

Keywords: Concept map; knowledge; assessment; instruction; web: graphed. 


\section{Introduction}

Research and development of new approaches and tools for education and performance support is rarely, if at all, amenable to sudden changes in pace and demand. Nevertheless, the ongoing worldwide pandemic has forced full-scale adoption of online learning at an accelerated pace. The uptake in the development of technologies supporting remote activities and work has somewhat facilitated the sudden move to online learning. However, this transition has proven to be much more challenging than expected and in dire need of tools and approaches to help assess information delivery, as well as knowledge building and retention. Although most of the traditional assessment methods, such as essays, quizzes, or tests, are still effective, they generate an incomplete picture and are seldom able to offer insights into the learner's mental schema underlying a specific cognitive process. Therefore, educators are always searching for better tools to help them gain deeper insights into how their students think and learn.

One such tool is the concept map, which can be used both stand alone or in conjunction with the more traditional assessment methods. Because of its ability to succinctly and efficiently represent the connected nature of one's domain knowledge, the use of concept maps has the potential to significantly enrich educators' understanding of their students' learning. When implemented properly, the use of concept maps has the power to increase instructional efficiency and efficacy.

Unfortunately, concept maps have a significant drawback. Their evaluation and assessment are notoriously difficult to automate. Traditionally, concept maps are evaluated qualitatively, using scoring rubrics or other structured means, which is a laborious qualitative process (Jonassen, 2006; McLinden, 2017). Notably, attempts have been made to use mathematical and computational tools to evaluate concept maps (e.g., Taricani \& Clariana, 2006), but the difficulties posed by the high variability of expression in building the concept maps has prevented the use of these methods in real applications to become reality. Advances in programming languages, computational tools, and mobile technologies may now help address some of the previous barriers inherent to the use of concept maps in education.

For this purpose, we have designed and developed a web-based application, graphed (https://graphed.igiresearch.com), mostly to aid instructors in implementing concept maps as part of instruction. In this paper we introduce the first version of this application which, in addition to its use in the classroom, it was also designed to be a platform for data collection, for both usability testing and to support the evaluation of existing software, libraries, and computational avenues as tools for automated concept map assessment. 


\section{Background}

Concept maps, also known as semantic networks, are well-established knowledge elicitation tools, utilized to express the connected nature of one's knowledge within a domain (Jonassen, 2006). Their origins can be traced back to Ausubel's (Ausubel et al., 1968) hierarchical memory theory and Deese's (Deese, 1965) associationist memory theory, which eventually converged towards the concept of concept maps as representations of one's knowledge. The term concept map was coined by Novak \& Govin (Novak \& Gowin, 1984). The idea that underlies the use of concept maps assumes that understanding any topic or subject requires people to form relationships between the various concepts and constructs relevant to the domain. Structural knowledge (why), which is what concept maps help elicit, is what connects the declarative (what) and procedural (how) knowledge together (Jonassen, 2006; Lee \& Murcia, 2013). Therefore, the elicitation of people's understanding of these relationships can be useful in assessing their comprehension of the domain with less subjectivity than other methods, such as written statements.

There is a wealth of research conducted on the implementation of concept maps for individual instruction. Studies have highlighted their value when used as a tool for consolidation of knowledge, aid to writing assignments, support for critical thinking, mediator of interaction between students, or assessment of structural knowledge (Cañas et al., 2003, McLinden, 2017). Unfortunately, the use of concept maps in large groups' instruction is hindered by their known difficulty of assessment. So far, the best way to evaluate concept maps is still primarily a qualitative process; the analysis and presentation of concept maps has not changed significantly since their inception (McLinden, 2017). Over the years, efforts have been made to deal with the wide variability in defining a concept map for the same knowledge domain, such as using similarity flooding algorithms (Marshall et al., 2006) or genetic algorithms (Rocha et al., 2004). Attempts to apply numerical and computational approaches to the evaluation and comparison of concept maps have also been made (Rocha et al., 2004), but the process has limitations (Limongelli et al., 2017; Siew, 2018; Siew et al., 2019). McLinden (2017) offers a summary of promising numerical and computational methods covering multidimensional scaling analysis and clustering algorithms using the $\mathrm{R}$ language and social network analysis. Cañas et al. (2018) have extended CMap to work over the web. Pathfinder networks and latent semantic analysis was also proposed for concept map analysis (Clariana et al., 2006; Koul et al., 2005; Taricani \& Clariana, 2006).

\section{Rationale \& Purpose}

The published literature suggests that most, if not all these attempts have merit, but it also notes that more research is needed to make them useful in practice. Searching for concept mapping tools available today returns many choices; for example a Google Scholar search of 
"concept map" yielded almost 60,000 results. Unfortunately, the concept mapping applications we could find across the web are either too generic, proprietary, or too complicated to use at scale in the classroom. Furthermore, most of them are designed to support the free-form development of concept maps and do not seem to offer usable computer-based concept map analysis and assessment, beyond basic metrics.

Based on the current state of knowledge our intentions follow a more practical path: use existing software, libraries, and computational approaches to support the use of concept mapping techniques in the classroom, for both instruction and assessment, while gathering usability data that can further advance our understanding of the tool, and to generate ideas for further development of the software.

\section{Design \& Development}

On the one hand, graphed does not place, by default, too many constraints on how the maps are represented, therefore allowing for significant flexibility and adaptability in use. On the other hand, its design offers ways for imposing constraints, to support a wider range of applications as both an educational tool and data collection platform.

At this stage, the application was built to support the basic representation of a concept map. Within graphed, concepts are represented as nodes, understood as "perceived regularity in events or objects, or a record of events or objects, designated with a label" (Cañas et al., 2003). Connectors are represented as labeled (usually written as verbs) directed arrows, linking the concepts together, expressing the relationships between these concepts. Together, two concepts and a labeled arrow form a proposition that represents a meaningful statement about the relationship they describe. In the end, the map should be able to tell a story.

While intuitive to use, there is a learning curve associated with successfully deploying this app in an online learning environment. Due to its many benefits, we opted for an audio-visual approach in guiding first-time users (either instructors or students), in the form of short explanatory videos and step-by-step demonstrations. A pilot testing of the training modules in a real classroom showed that students (graduate level course) overwhelmingly preferred videos to written instructions. Nevertheless, a comprehensive user guide in PDF format is currently under development, as it is easier updating as the development of the application continues.

To date, two of the primary user-related areas of graphed development have been focused on fine-tuning the graphical interface, and in deploying the collaboration tools which allow teams of students to work together to construct concept maps. As we have stated previously, feasible technologies and algorithms for automatically interpreting concept maps are in need of development. graphed can offer researchers in this field access to comprehensive data 
about the students' journey in developing concept maps, thus positioning itself as a research tool, in addition to being an instructional tool. The development of graphed as a web application gives us the ability to add functionality as we gain understanding, without disrupting the instances where the app has been already implemented as an instructional tool.

The design and development of the application is based on just a few foundational considerations:

- Mapping process should be made as simple as possible, without hurting the richness of expression.

- The application should be available everywhere the web can reach and on any device of any size. So far, the application is working, but the user experience on smallscreen mobile devices still needs improvement.

- Offer flexibility in defining the task. To do this, the application (so far) offers the following options:

- Constrained Concept Maps, for which the set of concepts has been predefined. The task would only require connecting these concepts with named connectors, selected from a predefined set, to form propositions.

- Open Concept Maps, for which there is no predefined set of concepts. The task requires the definition and naming of concepts and the construction of the connections between them.

- Availability of collaboration options. Currently, the application offers only two ways of working with concept maps:

- An individual mode, in which the users develop the maps by themselves, without seeing other users' maps.

- A collaborative mode, in which the user can both explore and work on each other's maps.

One of the guiding principles for the development of this first version of the application was to explore ways to limit variability to allow us to test as many evaluation algorithms as possible. Therefore, we decided to allow instructors to predefine the concepts to be used in a map (Constrained Concept Maps), which removes the variability generated by using different names to define the same concept or construct. We also decided to use a standardized list of connector names (Jonassen, 2006, p. 109), which allows us to limit the variability introduced by open-ended connector naming. The use of a specific list of connector names offers the added benefit of enabling the ability to build a connector weight and equivalence map that can help refine the analysis further. Should the need arise, this list can be extended. 
From a technical perspective, we chose to use Python for the server-side programming language with CherryPy for the web framework, visjs for the concept map visualization in the browser, and PostgreSQL for the database back-end. We chose Python because it arguably offers the most comprehensive set of libraries for data analysis as well as great interoperability with other server-side languages, such as $\mathrm{R}$, that may be used in the future. Visjs was chosen because of its visualization and editing capabilities, its compatibility with browser engines, and data representation. PostgreSQL was chosen for its ability to use document storage (JSONB) inside a relational database, which offers us the ability to store map data in a compact, searchable format.

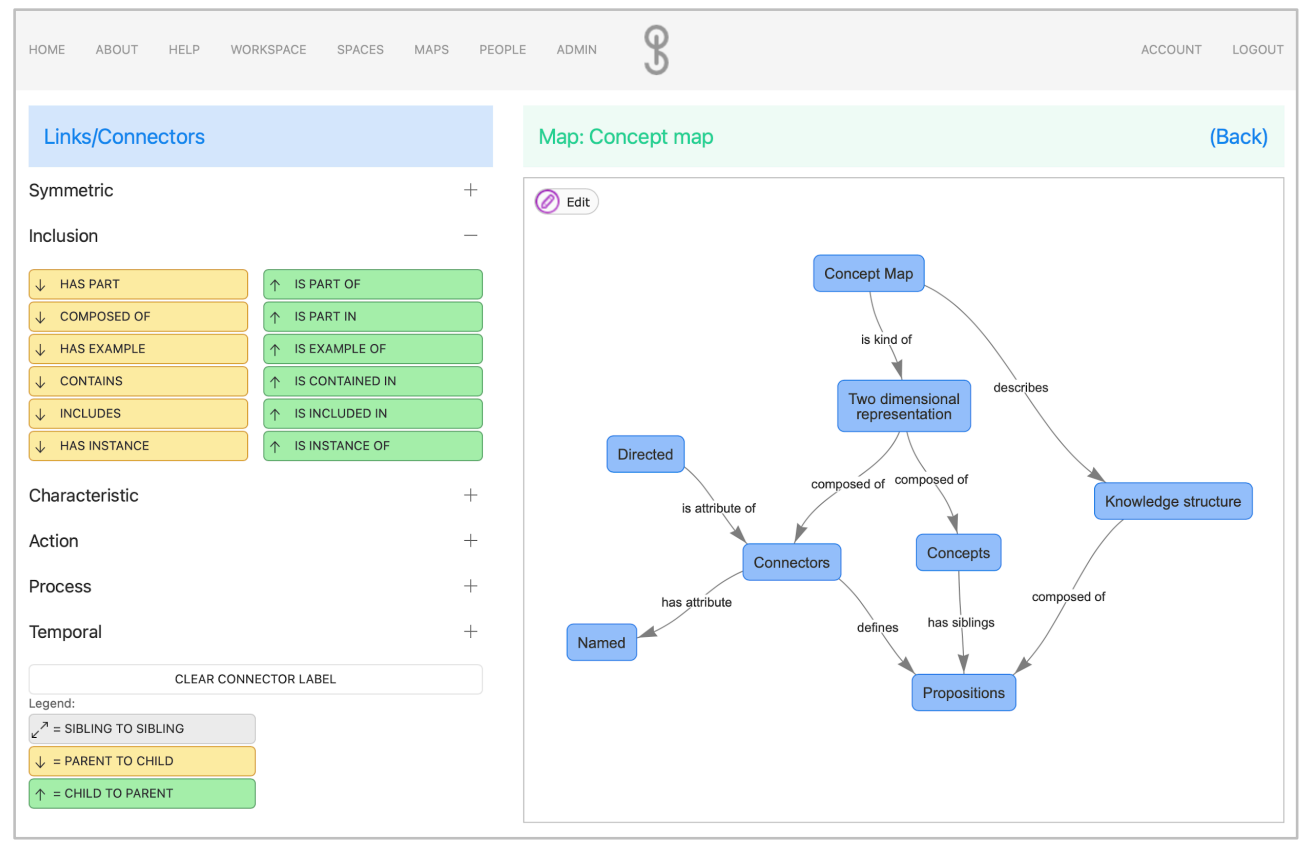

Figure 1. graphed. Concept map builder interface

In the Spring 2021 semester, the graphed web application has been used to support a graduate level course in social sciences. We used this opportunity to collect the first round of studentgenerated concept maps and application usability data. Simultaneously, we are evaluating existing tools that we could implement to assist instructors with using concept maps for the assessment of students' academic achievement. We purposefully chose a small class (nine students) to allow for richer feedback from the users and close interaction with the instructor.

The use of graphed has been closely integrated with the course syllabus, as a supporting tool for instruction and assessment. The didactic approach has also been slightly altered to focus more on relevant concepts and constructs for the domain, the relationships between them, 
and how they all can be tailored to contribute to the development and justification of a comprehensive research project.

So far, we can only report on implementation and preliminary results. The first step was to train the students on the principles and practice of concept mapping and use of the website. Video tutorials and personal guidance were both available. They were also encouraged to explore the website and build concept maps on their own. Next, the students were asked to build a constrained concept map using a predefined set of 23 concepts covered in class. For this activity the instructor chose to disable collaboration between students within the shared space. The final step was to conduct an informal virtual focus group where the students were asked to offer feedback about the software and the task. The students recognized that the concept mapping activity required them to think deeper about the relationships between the concepts and their organization within the field, with one suggesting that it would be helpful o build a map at the beginning of the class, and one at the end so that they can see the progress. One comment stood out: a student who is also a teacher noted that she would see herself using this website in her classroom. The students also made suggestions for the improvement of the application. They ranged from improvements to the interface to added functionality. The difficulty of use on small screen mobile devices was also brought up.

\section{Future Directions}

This first version of graphed is an early foray into the quest to find an intuitive and scalable solution to facilitate the inclusion of concept maps as an educational tool, one that can be seamlessly used in both online as well as in-person instruction environments. The future development of the software solution will be data driven. Qualitative research will gather both instructors' and student's feedback on implementing concept maps as part of a course, and will be used to further tweak both the user interface and the usability of the web app. It will also be used in assessing the concept maps to offer a base of comparison between humans and machines. Quantitative research will focus on the development of specific metrics to analyze concept maps and evaluate similarities and differences between them, as well as modalities in which these metrics can be standardized and deployed as part of an automated assessment and evaluation algorithm within graphed. Planned experimental research will also study knowledge acquisition and retention associated with the use of concept maps in the classroom, as it relates to the new assessment approaches.

\section{References}

Ausubel, D. P., Novak, J. D. \& Hanesian, H. (1968). Educational Psychology: A Cognitive View. Holt, Rinehart \& Winston. 
Cañas, A. J., Coffey, J. W., Carnot, M. J., Feltovich, P., Hoffman, R. R., Feltovich, J. \& Novak, J. D. (2003). A Summary of Literature Pertaining to the Use of Concept Mapping Techniques and Technologies for Education and Performance Support.

Cañas, A., Carff, R., \& Lott, J. (2018, September 28). eCMap: An Embeddable Web-Based Concept Map Editor.

Clariana, R. B., Koul, R. \& Salehi, R. (2006). The Criterion-Related Validity of a ComputerBased Approach for Scoring Concept Maps. International Journal of Instructional Media, 33, 317-325.

Deese, J. (1965). The structure of associations in language and thought. Johns Hopkins Press.

Jonassen, D. H. (2006). Modeling with technology: Mindtools for conceptual change (3rd ed). Pearson Merrill Prentice Hall.

Koul, R., Clariana, R. B. \& Salehi, R. (2005). Comparing Several Human and ComputerBased Methods for Scoring Concept Maps and Essays. Journal of Educational Computing Research, 32(3), 227-239. https://doi.org/10.2190/5x9y-0etn-213u-8fv7

Lee, C. B., \& Murcia, K. (2013). Problem Solving for Conceptual Change. In J. M. Spector, B. B. Lockee, S. E. Smaldino, M. C. Herring, \& J. M. Spector (Eds.), Learning, problem solving, and mind tools: Essays in honor of David H. Jonassen (pp. 195-213). Routledge.

Limongelli, C., Sciarrone, F., Lombardi, M., Marani, A. \& Temperini, M. (2017). A framework for comparing concept maps. 1-6. https://doi.org/10.1109/ithet.2017.8067818

Marshall, B., Chen, H. \& Madhusudan, T. (2006). Matching knowledge elements in concept maps using a similarity flooding algorithm. Decision Support Systems, 42(3), 1290-1306. https://doi.org/10.1016/j.dss.2005.10.009

McLinden, D. (2017). And then the internet happened: Thoughts on the future of concept mapping. Evaluation and Program Planning, 60, 293-300. https://doi.org/10.1016/j.evalprogplan.2016.10.009

Novak, J. D. \& Gowin, D. B. (1984). Learning How to Learn. Cambridge University Press. https://www.cambridge.org/core/books/learning-how-tolearn/D4E082D454735D8CC7FEDADFA25A3B99

Rocha, F. E. L. da, Jr, J. V. da C. \& Favero, E. L. (2004). A New Approach to Meaningful Learning Assessment Using Concept Maps: Ontologies and Genetic Algorithms. In A. J. Cañas, J. D. Novak \& F. M. Gonzálz (Eds.), Proc. of the First Int. Conference on Concept Mapping.

Siew, C. S. Q. (2018). Using network science to analyze concept maps of psychology undergraduates. Applied Cognitive Psychology, 33(4), 662-668. https://doi.org/10.1002/acp.3484

Siew, C. S. Q., Wulff, D. U., Beckage, N. \& Kenett, Y. (2019). Cognitive Network Science: A review of research on cognition through the lens of network representations, processes, and dynamics. https://doi.org/10.31234/osf.io/eu9tr

Taricani, E. M. \& Clariana, R. B. (2006). A Technique for Automatically Scoring OpenEnded Concept Maps. Educational Technology Research and Development, 54(1), 6582. https://doi.org/10.1007/s11423-006-6497-z 